\title{
CONTINUOUS FUNCTIONS FROM A CONNECTED LOCALLY CONNECTED SPACE INTO A CONNECTED SPACE WITH A DISPERSION POINT
}

\author{
C. A. COPPIN
}

\begin{abstract}
For $T_{2}$ spaces, it is shown that any continuous function from a connected locally connected space into a connected space with a dispersion point is a constant.
\end{abstract}

The purpose of this note is to present a result of connected spaces with a dispersion point which seems to have gone unnoticed or is part of the folklore of mathematics, and then we would like to make a remark. There are many examples of connected spaces with a dispersion point, a classic one given by Knaster and Kuratowski [1] and for others, see [2], [3], [4]. For terminology, see [6]. All spaces are $T_{2}$.

THEOREM. Any continuous function from a connected locally connected space into a connected space with a dispersion point is a constant.

Proof. Suppose $Y$ is a connected space with a dispersion point $p$ and $f$ is a continuous function from the locally connected space $X$ into $Y$.

Assume that $f$ is not constant. This means that $f(X)$ is a nondegenerate connected subset of $Y$ and therefore contains $p$.

By assumption $f(X)-\{p\} \neq \varnothing$, and therefore the set $G=f^{-1}(Y-\{p\})$ is not empty and different from $X$. Denote by $U$ any component of $G$. Since $X$ is locally connected and $G$ open, then $U$ is open. We shall show that $U$ is closed (this will contradict the assumption of connectedness of $X$ ).

Since $U$ is connected, so is $f(U)$, and since $f(U) \subset f(G) \subset Y-\{p\}$, we have $p \notin f(U)$. It follows that $f(U)$ is a single point $q \neq p$.

Now to show that $U$ is closed, let $x \in \bar{U}$. Hence $f(x) \in \mathrm{Cl}(f(U))$. But, since $f(U)=\{q\}$, we have $\mathrm{Cl}(f(U))=\{q\}$ and hence $f(x)=q \neq p$, i.e. $x \in f^{-1}(Y-\{p\})=G$.

Denote by $W$ the component of $G$ which contains $x$. Thus $x \in \bar{U} \cap W$ and it follows that $W=U$ since $W$ and $U$ are open sets, either disjoint or identical. Hence $x \in U$. Thus $U$ is closed.

Received by the editors November 24, 1970.

AMS 1970 subject classifications. Primary 54C05, 54D05.

Key words and phrases. Connected space, continuous function, dispersion point, locally connected space.

(c) American Mathematical Society 1972 
REMARK. In the original manuscript presented to the editors, we posed the question: Does there exist a nondegenerate connected space $Y$ without a dispersion point such that any continuous function from a connected locally connected space into $Y$ is constant? By methods similar to the above, Mr. J. Krasinkiewicz has shown that a widely connected space has the required property. A connected space $Y$ is said to be widely connected if each nondegenerate connected subset of $Y$ is dense in $Y$. P. M. Swingle [5] has shown the existence of such a space in the plane.

The author wishes to thank the referee for his extensive help.

\section{REFERENCES}

1. B. Knaster and C. Kuratowski, Sur les ensembles connexes, Fund. Math. 2 (1921), 206-255.

2. J. Martin, A countable Hausdorff space with a dispersion point, Duke Math. J. 33 (1966), 165-167. MR 33 \#699.

3. G. G. Miller, Countable connected spaces, Proc. Amer. Math. Soc. 26 (1970), 355-360. MR 41 \#7610.

4. P. Roy, A countable connected Urysohn space with a dispersion point, Duke Math. J. 33 (1966), 331-333. MR 33 \#4887.

5. P. M. Swingle, Two types of connected sets, Bull. Amer. Math. Soc. 37 (1931), 254.

6. S. Willard, General topology, Addison-Wesley, Reading, Mass., 1970.

Department of Mathematics, University of Dallas, Irving, TeXas 75060 